\title{
USO DE SUBSTRATOS ORGÂNICOS PARA PRODUÇÃO DE MUDAS DE ALFACE
}

\author{
Israel Martins Pereira ${ }^{1}$ \\ Rebyson Bissaco Guidinelle ${ }^{2}$ \\ Alex Justno Jacarias ${ }^{3}$ \\ Júlio Cesar Gradice Saluci ${ }^{4}$ \\ Mário Euclides Pechara da Costa Jaeggi ${ }^{5}$ \\ Ronald Martins Pereira Junior ${ }^{6}$ \\ Maxwel Rodrigues Nascimento ${ }^{7}$ \\ Rogério Rodrigues Range ${ }^{8}$ \\ Manuela Arruda Contarine ${ }^{9}$ \\ Wallace Luís de Lima ${ }^{10}$
}

Resumo: O trabalho teve como objetivo avaliar o efeito de substratos orgânicos na produção de mudas de alface. O delineamento experimental utilizado foi o inteiramente casualizado (DIC) disposto em esquema fatorial 3x2, sendo constituídas por três substratos duas cultivares se alface (Regina e Mônica). Em bandejas de isopor que continham os diferentes substratos: Comercial, Vermicomposto e Serapilheira. Estudaram-se as variáveis: a) avaliados foram massa fresca parte aérea (MFPA) obtida a partir da pesagem da parte aérea da plântula, b) massa fresca sistema radicular (MFSR), obtido pelo peso da raiz lavada sobre uma peneira de malha fina usando água corrente, c) massa fresca total (MFT) obtida através da soma da MFPA+MFSR e número de folhas (NFT), contando-se as unidades de folhas, foram avaliadas após 25 dias emergência das sementes. Obtendo-se valores significativos, percebeu-se que o substrato de Vermicomposto se sobressaiu com resultado melhor que o composto Comercial e Serapilheira.

Palavras-chave: Lactuca sativa L; Cultivares; Emergência.

\footnotetext{
1 Tecnologia em Cafeicultura/Instituto Federal do Espirito Santo - Campus de Alegre, Brasil. E-mail: israelmartins80@gmail.com.

2 Tecnologia em Cafeicultura/Instituto Federal do Espirito Santo - Campus de Alegre, Brasil. E-mail: rebysonguidinelle@gmail.com.

3 Tecnologia em Cafeicultura/Instituto Federal do Espirito Santo - Campus de Alegre, Brasil. E-mail: alexjustino12@gmail.com.

4 Tecnologia em Cafeicultura/Instituto Federal do Espirito Santo - Campus de Alegre, Brasil. E-mail: juliosaluci@gmail.com.

5 Produção Vegetal/Universidade Estadual Norte Fluminense Darcy Ribeiro, Centro de Ciências e Tecnologias Agropecuárias, Brasil. E-mail: mariopechara@hotmail.com.

6 Engenharia Agronômica/Universidade Federal do Espírito Santo, Centro de Ciências Agrárias - Campus de Alegre, Brasil. E-mail: ronal_martins_pereira@hotmail.com.

7 Produção Vegetal/Universidade Estadual Norte Fluminense Darcy Ribeiro, Centro de Ciências e Tecnologias Agropecuárias, Brasil. E-mail: maxwel.m88@gmail.com.

8 Engenharia agrícola/Universidade Federal de Lavras, Brasil. E-mail: rogeriorr7@hotmail.com.

9 Tecnologia em Cafeicultura/Instituto Federal do Espirito Santo - Campus de Alegre, Brasil. E-mail: manucontarine@gmail.com.

${ }^{10}$ Professor D.Sc. Agronomia - Ciência do Solo no Instituto Federal do Espirito Santo - Campus de Alegre, Brasil. E-mail: wallace@ifes.edu.br.
} 